\title{
Use of special symbols
}

A bold typeface within an entry shows a cross-reference to another entry. Major cross-references are often at the end of an entry.

Italics are used to represent an English example or word used for illustration.

Bold italics, when they occur as part of an example, are meant to highlight or emphasize that part of the example.

Bold italics also refer to a verb as if in a dictionary, where the different verb forms are irrelevant. (For example, look means look/looks/looked/looking.)

A swung dash indicates a relation between alternative forms of the same basic word or pattern, for example: boy boys.

( ) Round brackets in examples indicate something which is optional, that is, can be omitted or ignored.

[ ] For clarity, square brackets are sometimes used in examples to signal the boundaries of a major constituent, usually a clause.

I Vertical lines in examples are sometimes used to separate main elements of a clause, for example: subject, verb phrase and object.

* An asterisk before an example indicates that the example is unacceptable as a piece of English.

Note

The terminology in this book is mainly based on that of Randolph Quirk et al. (1985): A Comprehensive Grammar of the English Language - see the Introduction. 